\title{
A NEW EMBEDDING SCHEME FOR GROUPS AND SOME APPLICATIONS
}

\author{
VIATCHESLAV N. OBRAZTSOV
}

(Received 23 April 1994; revised 24 March 1995)

Communicated by R. Howlett

\begin{abstract}
In this paper a scheme of an 'economical' embedding of an arbitrary set of groups without involutions in an infinite group with a proper simple normal subgroup is presented. This scheme is then applied to construction of groups with new properties.
\end{abstract}

1991 Mathematics subject classification (Amer. Math. Soc.): 20F05, $20 \mathrm{~F} 06$.

\section{Main result and its corollaries}

Many properties of a group are closely connected with the structure of its subgroups. In [7] was proved a theorem on embeddability of every at most countable group $A$ without involutions in a simple 2-generator group in which every proper subgroup is either a cyclic group or contained in a subgroup conjugate to $A$, and an embedding scheme of an arbitrary set of groups without involutions in a simple group $G$ with 'well-described' lattice of subgroups was established in [8]. But for the solution of some group-theoretical problems, we need a generalization of these embedding schemes giving a group $G$ with a proper normal subgroup.

Let $\left\{G_{i}\right\}_{i \in I},|I|>1$, be an arbitrary set of non-trivial groups without involutions. We denote by $\Omega^{1}$ the free amalgam of the groups $G_{i}, i \in I$, that is, the $\operatorname{set} \bigcup_{i \in I} G_{i}$ with $G_{i} \cap G_{j}=1$ whenever $i \neq j$. We say that the mapping $g: \Omega^{1} \rightarrow G$ is an embedding of $\Omega^{1}$ into $G$ if it is injective and its restriction to every $G_{i}$ is a homomorphism.

Let $\Omega=\Omega^{1} \backslash\{1\}=\left\{a_{j}, j \in J\right\}$. Then as in [8], a mapping $f: 2^{\Omega} \backslash\{\emptyset\} \rightarrow 2^{\Omega}$ is called generating on the set $\Omega$ if the following conditions hold:

(1) if $C \subseteq G_{i}$ for some $i \in I$ then $f(C)=\operatorname{gp}\{C\} \backslash\{1\}$;

(c) 1996 Australian Mathematical Society 0263-6115/96 \$A2.00+0.00 
(2) if $C$ is a finite subset of $\Omega$ and $C \nsubseteq G_{i}$ for each $i \in I$, then $f(C)=B$, where $B$ is an arbitrary finite or countable subset of $\Omega$ such that $C \subseteq B$ and if $D$ is a finite subset of $B$, then $f(D) \subseteq B$;

(3) if $C$ is an infinite subset of $\Omega$ and $C \nsubseteq G_{i}$ for each $i \in I$, then $f(C)=$ $\bigcup_{A \in T} f(A)$, where $T$ is the set of all finite subsets of $C$.

For example, a generating mapping $f$ on $\Omega$ can be defined in the following way: if $C \in 2^{\Omega} \backslash\{\emptyset\}$ and $C=\bigcup_{i \in I} C_{i}$, where $C_{i}=C \cap G_{i}, i \in I$, then $f(C)=$ $\left(\bigcup_{i \in I} \operatorname{gp}\left\{C_{i}\right\}\right) \backslash\{1\}$.

We denote by $G(1)$ the free product of groups $G_{i}, i \in I$. A group $G$ having the presentation

$$
G=\langle G(1) \mid R=1 ; R \in D\rangle
$$

is called (diagrammatically) aspherical ((diagrammatically) atoroidal) if every diagram on the sphere (torus) over (1.1) is either non-reduced or consists entirely of 0 -cells. (All necessary information about diagrams can be found in [10].)

Let $G=\operatorname{gp}\{\Omega\}, f$ an arbitrary generating mapping on $\Omega$. We say that $X$ is a minimal word of a group $G$ if it is follows from $X=Y$ in $G$ that $|X| \leq|Y|$, where $|Z|$ denotes the length of the word $Z$. Let $W$ be the set of all non-empty words over the alphabet $\Omega$ written in the normal form, that is, every element $X$ in $W$ is written in the form $X_{1} \ldots X_{k}$, where each $X_{l}, 1 \leq l \leq k$, is a non-trivial element of $G_{\mu(l)}, \mu(l) \in I$, and $\mu(l) \neq \mu(l+1)$ for $l=1, \ldots, k-1$. Then a mapping $F: 2^{W} \backslash\{\emptyset\} \rightarrow 2^{\Omega}$ is defined in the following way: if $C \subseteq W$ and $C \neq \emptyset$ then let $V$ be the set of all letters occuring in the expressions of words of $C$. Then we set $F(C)=f(V)$.

The main result of this paper is the following embedding scheme:

THEOREM A. Let $m$ be a sufficiently large odd number or $m=\infty, g_{i}: G_{i} \rightarrow H$ a set of arbitrary homomorphisms of groups with kernels $N_{i}, i \in I$, such that a system of subgroups $\left\{g_{i}\left(G_{i}\right)\right\}_{i \in I}$ generates $H$, let $\left\{N_{j}\right\}_{j \in I_{1}}, I_{1} \subseteq I$, be the set of nontrivial groups of the set $\left\{N_{i}\right\}_{i \in I}, \Omega_{1}^{1}$ the free amalgam of the groups $N_{j}, j \in I_{1}$, and let $f$ be an arbitrary generating mapping on $\Omega$ such that $f(C) \cap \Omega_{1}^{1} \neq \emptyset$ if $C \nsubseteq G_{i}$ for each $i \in I$. If $\left|I_{1}\right|>1$ then the free amalgam $\Omega^{1}$ of the groups $G_{i}$ can be embedded in an aspherical atoroidal group $G=\operatorname{gp}\{\Omega\}$ with the following properties:

(1) the free amalgam $\Omega_{1}^{1}$ is embedded in a normal simple infinite subgroup $L$ of $G$ such that $G / L \cong H$;

(2) if $X \in L$ and $X$ is not conjugate in $G$ to an element of one of the groups $G_{i}, i \in I$, then either $X$ is equal to a power of an element $Y$, where $Y$ is of infinite order and whose homomorphic image in $H$ has even order, or $X$ is of order dividing $m$ (of infinite order in the case $m=\infty$ );

(3) Aut $L \cong G$ (and so Out $L \cong H$ ) and if $g \in G_{i} \backslash \Omega_{1}^{1}, i \in I$, then the mapping $g: L \rightarrow g^{-1} L g$ is a regular automorphism of $L$ (that is, $g(a)=a$ if and only if 
$a=1)$ if and only if there is no $c \in G_{i} \cap \Omega_{1}$, where $\Omega_{1}=\Omega_{1}^{1} \backslash\{1\}$, such that $[g, c]=1$;

(4) every subgroup $M$ of $G$ is either a cyclic group or $M \cap L=1$ and the homomorphic image of $M$ in $H \cong G / L$ has an element of infinite order, or $M$ is conjugate in $G$ to an extension $G_{C, H^{\prime}}$ of a group $H^{\prime}$ by a normal subgroup $L_{C}$ (that is, $G_{C, H^{\prime}} / L_{C} \cong H^{\prime}$ ), where $H^{\prime} \leq H$ and if every element of $L_{C}$ is a minimal word of $G$, then $C=F\left(L_{C} \backslash\{1\}\right)$ or $C=\emptyset$ in the case $L_{C}=\{1\}$;

(5) $L_{C} \leq R_{C} \cap L$, where $R_{C}=\operatorname{gp}\{C\}, C \in 2^{\Omega} \backslash\{\emptyset\}$ or $R_{C}=\{1\}$ in the case $C=\emptyset$, and if $C \nsubseteq G_{i}$ for each $i \in I$, then $L_{C}=R_{C} \cap L$ and $G_{C, H^{\prime}} \leq R_{C}$;

(6) if $C \nsubseteq G_{i}$ for each $i \in I$, then for each $a \in f(C) \cap \Omega_{1}, L_{C}=\operatorname{gp}\left\{b a b^{-1}, b \in\right.$ $f(C)\}$ (in particular, $L=\mathrm{gp}\left\{b a b^{-1}, b \in \Omega\right\}$, where $a$ is an arbitrary element of $\left.\Omega_{1}\right)$;

(7) if $X$ is a minimal non-trivial word of the group $G$, then $X \in R_{C}$ if and only if $F(\{X\}) \subseteq f(C)$

(8) if $\left\{G_{j}\right\}_{j \in J}, J \subseteq I$, is a set of all groups having non-trivial intersections with a subgroup $R_{C}$ of $G$ and $X \in Z^{-1} R_{C} Z$, where $|Z|$ is the minimal among all words in $R_{C} Z$ and $G_{j} Z$ for each $j \in J$, then $F(\{Z\}) \subseteq F(\{X\})$;

(9) if $C \nsubseteq G_{i}$ for each $i \in I, M$ is a subgroup of $G$ in which every element is a minimal word of $G$, then $\operatorname{gp}\left\{L_{C}, M\right\} \cap L=L_{C_{1}}$, where $C_{1}=F(C \cup(M \backslash\{1\}))$;

(10) if $H=G_{s}$ for some $s \in I$ and the homomorphism $g_{j}: G_{j} \rightarrow H$ is trivial for each $j \in I \backslash\{s\}$, then $G$ is the semidirect product of $H$ and $L$.

The first corollary of Theorem A is devoted to the groups of outer automorphisms of simple infinite groups. Matumoto [5] proved that every group is isomorphic to the outer automorphism group of some group, and a scheme of an 'economical' embedding of an arbitrary set of groups without involutions in a simple complete group (that is, a group with trivial centre and no outer automorphisms) was established in [9]. Now we have

THEOREM B. Let $\left\{G_{i}\right\}_{i \in I},|I|>1$, be an arbitrary set of non-trivial groups without involutions, $H$ an arbitrary (in particular, trivial) group without involutions, $\Omega^{1}$ the free amalgam of the groups $H$ and $G_{i}, i \in I$, and let $f$ be an arbitrary generating mapping on $\Omega=\Omega^{1} \backslash\{1\}, m$ a sufficiently large odd number or $m=\infty$. Then the free amalgam $\Omega^{1}$ can be embedded in an aspherical atoroidal group $G=\operatorname{gp}\{\Omega\}$ with the following properties:

(1) the free amalgam of the groups $G_{i}$ is embedded in a simple normal infinite subgroup $L$ of $G$ and $G / L \cong H$;

(2) Out $L \cong H$ and for each $g \in H \backslash\{1\}$, g is a regular automorphism of $L$;

(3) every non-trivial subgroup of $L$ is a cyclic group of order dividing $m$ (an infinite cyclic group in the case $m=\infty$ ) or contained in a subgroup conjugate in 
$G$ to some $G_{i}$, or conjugate in $G$ to a subgroup $L_{C}=R_{C} \cap L$, where $C \in$ $2^{\Omega} \backslash 2^{H}, R_{C}=\operatorname{gp}\{C\}$, and $L_{C}=\operatorname{gp}\left\{b a b^{-1}, b \in f(C)\right\}$ for each $a \in f(C) \backslash H$.

PROOF. Let $g_{i}: G_{i} \rightarrow H$ be the trivial homomorphism for each $i \in I, g_{H}$ : $H \rightarrow H$ the natural isomorphism. Then a system $\left\{N_{i}\right\}_{i \in I}$ of non-trivial kernels of the homomorphisms $g_{H}$ and $g_{i}, i \in I$, is the same as the set of the groups $G_{i}, i \in I$, and hence Theorem A applies to $\Omega^{1}, f$ and $m$ and yields the required $G$.

If the condition 'a group $H$ has no involutions' is omitted, then the situation is more complicated.

THEOREM C. Let $\left\{G_{i}\right\}_{i \in I},|I|>1$, be an arbitrary set of non-trivial groups without involutions, $H=\mathrm{gp}\left\{h_{j}\right\}_{j \in J}$ an arbitrary (in particular, trivial) group, $n_{j}$ the order of $h_{j}$ in $H, j \in J$, let $\left\{S_{j}=\operatorname{gp}\left\{s_{j}\right\}\right\}_{j \in J}$ be a set of infinite cyclic groups, $\Omega^{1}$ the free amalgam of the groups $\left\{G_{i}\right\}_{i \in I}$ and $\left\{S_{j}\right\}_{j \in J}, \Omega_{1}^{1}$ the free amalgam of the groups $\left\{G_{i}\right\}_{i \in I}$ and $\left\{\operatorname{gp}\left\{s_{j}^{n_{j}}\right\}\right\}_{j \in J}$, where $\operatorname{gp}\left\{s_{j}^{n_{j}}\right\}=\{1\}$ if $n_{j}=\infty$, and let $f$ be an arbitrary generating mapping on $\Omega=\Omega^{1} \backslash\{1\}$ such that $f(C) \cap \Omega_{1}^{1} \neq \emptyset$ if $C \nsubseteq S_{j}$ for each $j \in J$. Then the free amalgam $\Omega^{1}$ can be embedded in an aspherical atoroidal group $G=\operatorname{gp}\{\Omega\}$ with the following properties:

(1) the free amalgam $\Omega_{1}^{1}$ is embedded in a simple normal infinite subgroup $L$ of $G$ and $G / L \cong H$;

(2) Out $L \cong H$;

(3) every non-trivial subgroup of $L$ is an infinite cyclic or contained in a subgroup conjugate in $G$ to some $G_{i}$, or conjugate in $G$ to a subgroup $L_{C}=R_{C} \cap L$, where $C \in 2^{\Omega} \backslash\{\emptyset\}, R_{C}=\operatorname{gp}\{C\}$, and $L_{C}=\operatorname{gp}\left\{b a b^{-1}, b \in f(C)\right\}$ for each $a \in f(C) \cap \Omega_{1}^{1}$.

PROOF. Let $g_{i}: G_{i} \rightarrow H$ be the trivial homomorphism for each $i \in I$, and for each $j \in J$, we define a homomorphism $g_{j}: S_{j} \rightarrow H$ by setting $g_{j}\left(s_{j}^{t}\right)=h_{j}^{t}, t \geq 1$. Then Theorem A applies to $\Omega^{1}, f$ and $m=\infty$ and yields the required $G$.

For countable groups, we have the following important corollary:

THEOREM D. Let $\left\{G_{i}\right\}_{i \in I},|I|>1$, be an at most countable set of non-trivial finite or countable groups without involutions, $H$ an arbitrary at most countable group, $m$ a sufficiently large odd number or $m=\infty$. Then the free amalgam of the groups $G_{i}$ can be embedded in a simple infinite group $L$ with the following properties:

(1) Out $L \cong H$, and if $H$ has no involutions then for each $g \in H \backslash\{1\}$, g is a regular automorphism of $L$; 
(2) every proper subgroup of $L$ is either an infinite cyclic group (a cyclic group of order dividing $m$ if $H$ has no involutions and $m<\infty$ ) or contained in a subgroup $\psi\left(G_{i}\right)$ for some $\psi \in$ Aut $L$ and $i \in I$.

ProOF. If $H$ has no involutions, then let $\Omega^{1}$ be the free amalgam of the groups $H$ and $G_{i}, i \in I$. If $H=\operatorname{gp}\left\{h_{j}\right\}_{j \in J}$ has involutions, then let $\Omega^{1}$ be the free amalgam of the groups $G_{i}, i \in I$, and of infinite cyclic groups $S_{j}=\operatorname{gp}\left\{s_{j}\right\}, j \in J$. In any case, we define a generating mapping $f$ on $\Omega=\Omega^{1} \backslash\{1\}$ in the following way: if $C \subseteq \Omega, C \nsubseteq G_{i}$ for each $i \in I$ and $C \nsubseteq H$ (and $C \nsubseteq S_{j}$ for each $j \in J$ in the second case), then $f(C)=\Omega$. Then Theorem B or Theorem $\mathrm{C}$ applies to $\Omega^{1}, m$ and this mapping $f$ and yields the group $G$ with the required normal subgroup $L$.

COROLLARY. Let $H$ be an arbitrary at most countable group. Then for any sufficiently large prime number $p$ or $p=\infty$, there exists a simple infinite group $L$ all of whose proper subgroups are infinite cyclic (cyclic groups of order $p$ if $H$ has no involutions and $p<\infty$ ) such that Out $L \cong H$, and if $H$ has no involutions then for each $g \in H \backslash\{1\}, g$ is a reqular automorphism of $L$.

PROOF. It is sufficient to take $G_{1}$ and $G_{2}$ to be cyclic groups of order $p$ and $L$ as the group in Theorem D for the set $\left\{G_{1}, G_{2}\right\}$ and $m=p$.

A group $G$ is called a $K$-group if its subgroup lattice is complemented, that is, for each $A \leq G$ there exists $B \leq G$ such that $A \cap B=1$ and $\operatorname{gp}\{A, B\}=G$. The following obvious remark will be used for proving results about $K$-groups: if $A, B \leq G, A \cap B=1$ and $\operatorname{gp}\{A, B\}=G$, then the groups $Z^{-1} A Z, Z^{-1} B Z$ satisfy these conditions for each $Z \in G$.

It is easy to see that a subgroup of a $K$-group is not, in general, a $K$-group, as the following example shows: $S_{4}$ is a $K$-group with cyclic subgroups of order 4 which are not $K$-groups. Further information on subgroups of $K$-groups is contained in

THEOREM E. Let $m$ be a sufficiently large odd number or $m=\infty,\left\{G_{i}\right\}_{i \in I},|I|>1$, an arbitrary set of non-trivial groups without involutions, $G_{0}$ a cyclic group of order $m$. Then the free amalgam $\Omega^{1}$ of the groups $G_{0}$ and $G_{i}, i \in I$, can be embedded in a simple infinite $K$-group $G=\operatorname{gp}\{\Omega\}$, where $\Omega=\Omega^{1} \backslash\{1\}$, such that every proper subgroup of $G$ is either a cyclic group of order dividing $m$ (an infinite cyclic group in the case $m=\infty$ ) or conjugate to a subgroup $R_{C}=\operatorname{gp}\{C\}$ for some $C \in 2^{\Omega} \backslash\{\emptyset\}$, where if $C \cap G_{0} \neq 1$ and $C \nsubseteq G_{0}$, then $G_{0} \subseteq C$, and $b \in R_{C} \cap G_{i}$, $i \in I \cup\{0\}$, if and only if $b \in C \cap G_{i}$.

ProOF. We set $H=\{1\}$ and define a generating mapping $f$ on $\Omega$ in the following way: if $C \subseteq \Omega, C \nsubseteq G_{0}$ and $C=\bigcup_{i \in I \cup\{0\}} C_{i}$, where $C_{i}=C \cap G_{i}, i \in I \cup\{0\}$, then

$$
f(C)=\left(G_{0}^{\prime} \cup \bigcup_{i \in I} \operatorname{gp}\left\{C_{i}\right\}\right) \backslash\{1\}
$$


where $G_{0}^{\prime}=G_{0}$ in the case $C_{0} \neq \emptyset$, for otherwise $G_{0}^{\prime}=\{1\}$. It remains to prove that the group $G$ taken as the group in Theorem $\mathrm{A}$ for $\left\{G_{i}\right\}_{i \in I \cup\{\mid\}}, m$ and the mapping $f$ is a $K$-group.

Let $M$ be a proper subgroup of $G, \Omega_{1}=\Omega \backslash G_{0}$ and $G_{0}=\operatorname{gp}\{a\}$. We consider the following cases:

(1) if $M=R_{C}$ and $\Omega_{1} \subseteq C$, then $G_{0} \cap C=\emptyset$ (since for otherwise $M=G$ ) and by Theorem A, $R_{C} \cap a^{-1} R_{\Omega_{1}} a=1$ and $\operatorname{gp}\left\{R_{C}, a^{-1} R_{\Omega_{1}} a\right\}=G$;

(2) if $M=R_{C}$ and there is $b \in \Omega_{1} \backslash C$, then it follows from Theorem A that $R_{C} \cap b^{-1} a^{-1} R_{\Omega_{1}} a b=1$ and $\operatorname{gp}\left\{R_{C}, b^{-1} a^{-1} R_{\Omega_{1}} a b\right\}=G$;

(3) if $M=\operatorname{gp}\{X\}$ is a cyclic group, then it is obvious that there is $Y \in G$ such that $a \in$ $F\left(\left\{Y^{-1} X Y\right\}\right)$, and by Theorem A, $M \cap Y R_{\Omega_{1}} Y^{-1}=1$ and $g p\left\{M, Y R_{\Omega_{1}} Y^{-1}\right\}=G$.

The proof of Theorem $\mathrm{E}$ is complete.

The following result is devoted to construction of $K$-groups having proper normal subgroups.

THEOREM F. If in the statement of Theorem A the map $g_{i}: G_{i} \rightarrow H$ is an isomorphism for some $i \in I$, the homomorphism $g_{j}: G_{j} \rightarrow H$ is trivial for each $j \in I \backslash\{i\}, H$ is a $K$-group and a generating mapping $f$ on $\Omega$ is defined in such a way that $F(H \cup\{a\})=\Omega$ for each $a \in \Omega_{1}$, then $G$ is a $K$-group.

PROOF. It follows from the statement of Theorem $F$ that $G$ is the semidirect product of $H$ and $L$. Let $M$ be a proper subgroup of $G$. Then the following cases are possible.

(1) If $M \cap L=1$ and $M_{1}$ is the homomorphic image of $M$ in $H$, then there is a subgroup $M_{2}$ of $H$ such that $M_{1} \cap M_{2}=1$ and $g p\left\{M_{1}, M_{2}\right\}=H$. Hence by Theorem $\mathrm{A}, M \cap M_{2} L=1$ and $\operatorname{gp}\left\{M, M_{2} L\right\}=G$.

(2) If $M \cap L \neq 1$ and $M \cap H=M_{1}$, then there is a subgroup $M_{2}$ of $H$ such that $M_{1} \cap M_{2}=1$ and $\operatorname{gp}\left\{M_{1}, M_{2}\right\}=H$. Then it follows from Theorem A that $M \cap M_{2}=1$ and $\operatorname{gp}\left\{M, M_{2}\right\} \supseteq \operatorname{gp}\{H, M \cap L\}=G$, as required.

By Theorem E, every group without involutions is a subgroup of some simple $K$-group. The situation with normal subgroups of $K$-groups is less clear. Emaldi asked in [4, problem 11.128] whether normal subgroups of $K$-groups are $K$-groups.

COROLLARY. There exists a $K$-group $G$ containing a normal simple infinite subgroup $L$ such that if $A, B \leq L$ and $\operatorname{gp}\{A, B\}=L$, then either $A=L$ or $B=L$.

PROOF. Let $m$ be a sufficiently large odd number or $m=\infty,\left\{G_{i}=\operatorname{gp}\left\{a_{i}\right\}\right\}_{i \geq 1}$ a set of cyclic groups of order $m$ (of infinite cyclic groups in the case $m=\infty$ ), $\Omega_{1}^{1}$ 
the free amalgam of the groups $\left\{G_{j}\right\}_{j \geq 3}$. Then Theorem E applies to the set $\left\{G_{j}\right\}_{j \geq 3}$ and $m$ and yields the $K$-group $H=\operatorname{gp}\left\{\Omega_{1}\right\}$, where $\Omega_{1}=\Omega_{1}^{1} \backslash\{1\}$, in which every proper subgroup is either a cyclic group of order dividing $m$ or conjugate to a subgroup $R_{C}=\operatorname{gp}\{C\}$ for some $C \in 2^{\Omega_{1}} \backslash\{\emptyset\}$, where if $C \cap G_{3} \neq 1$ and $C \nsubseteq G_{3}$, then $G_{3} \subseteq C$, and $a \in R_{C} \cap G_{j}, j \geq 3$, if and only if $a \in C \cap G_{j}$.

Let $\Omega^{1}$ be the free amalgam of the groups $H, G_{1}$ and $G_{2}$. A generating mapping $f$ on $\Omega=\Omega^{1} \backslash\{1\}$ is defined in the following way: if $C$ is a finite subset of $\Omega, C \nsubseteq H$ and $C \nsubseteq G_{i}, i=1,2$, then $k$ is the maximal index of letters of $\Omega^{\prime}=\left\{a_{i}\right\}_{i \geq 1}$ occurring in the expressions of words of $C$ (over the alphabet $\left.\Omega^{\prime}\right)$, then $f(C)=\left(\bigcup_{s \leq k} G_{s}\right) \backslash\{1\}$. Finally, if $C$ is an infinite subset of $\Omega, C \nsubseteq H$ and $C \nsubseteq G_{i}, i=1,2$, then $f(C)=\bigcup_{A \in T} f(A)$, where $T$ is the set of all finite subsets of $C$. Then Theorem $\mathrm{F}$ applies to the set $\left\{H, G_{1}, G_{2}\right\}$ (with trivial homomorphisms $g_{i}: G_{i} \rightarrow H, i=1,2$ ) and the mapping $f$ and yields the $K$-group $G$ with the simple infinite normal subgroup $L$.

Let for each $k \geq 2, \Omega_{k}^{1}$ be the free amalgam of the groups $\left\{G_{i}\right\}_{1 \leq i \leq k}$. Then by Theorem A, every proper subgroup of $L$ is either a cyclic group of order dividing $m$ or conjugate to a subgroup $S_{k}$ consisting of all minimal words $T$ of $L$ with $F(\{T\}) \subseteq$ $\Omega_{k}^{1}, k \geq 2$.

Let $A$ and $B$ be proper subgroups of $L$. For each minimal word $D$ of $L$, we denote by $M(D)$ the maximal index of letters occurring in the expression of $D$ (over the alphabet $\Omega^{\prime}$ ). Assume first that $A=\operatorname{gp}\{X\}$ and $B=\operatorname{gp}\{Y\}$, where $X$ and $Y$ are minimal words in $L$. Then it follows from Theorem A that $\operatorname{gp}\{A, B\} \leq$ $S_{k}$, where $k=\max (M(X), M(Y), 2)$. We now consider the second case when $A=Z^{-1} S_{k} Z, B=\operatorname{gp}\{X\}$, where $Z, X$ are minimal words in $L$. Then it follows from Theorem A that $\operatorname{gp}\{A, B\} \leq S_{t}$, where $t=\max (k, M(Z), M(X))$. The case when $A=\operatorname{gp}\{X\}, B=Z^{-1} S_{k} Z$ can be considered in a similar way. Finally if $A=Z_{1}^{-1} S_{k} Z_{1}, B=Z_{2}^{-1} S_{l} Z_{2}$ and $Z_{1}, Z_{2}$ are minimal words in $L$, then by Theorem $\mathrm{A}, \operatorname{gp}\{A, B\} \leq S_{t}$, where $t=\max \left(k, l, M\left(Z_{1}\right), M\left(Z_{2}\right)\right)$. This completes the proof of the corollary.

A group $G$ is called normally factorized if for each normal subgroup $A$ of $G$ there is $B \leq G$ such that $A \cap B=1$ and $A B=G$. It is obvious that every $K$-group is normally factorized. Moreover, these conditions coincide in some classes of groups, in particular, in the class of all soluble groups (Napolitani [6]), and in [3] it was noted that there were no examples to show that these conditions were distinct.

COROLLARY 2. The group $L$ in Corollary 1 provides an example of a simple (and hence normally factorized) group which is not a $K$-group.

The following result is connected with a question about Frattini subgroups. The Frattini subgroup $\Phi(G)$ of a group $G$ is the intersection of all the maximal subgroups 
of $G(\Phi(G)=G$ when $G$ has no maximal subgroups). In [2] and [7] were constructed countable simple groups without maximal subgroups. Of course, for each such group $G, \Phi(G)$ is a simple group. In his report at the Conference on Group Theory (Trento, Italy, 1993) J. Wiegold asked about the existence of a finitely generated group $G$ with non-trivial simple Frattini subgroup $\Phi(G)$.

THEOREM G. Let $H$ be an arbitrary periodic or abelian group with $d(H)=k, k \geq$ 2 , where $d(H)$ is the minimal number of generators of $H$, and let $s$ be a sufficiently large odd number or $s=\infty$. Then there exists a $k$-generator group $G$ such that

(1) $G$ has a normal simple infinite subgroup $L$ such that all proper subgroups of $L$ are infinite cyclic (cyclic groups of order dividing $s$ if $H$ has no involutions and $s<\infty)$ and $G / L \cong H$;

(2) every non-cyclic subgroup of $G$ contains $L$;

(3) $\Phi(G)$ is isomorphic to an extension of the group $\Phi(H)$ by $L$ (that is, $\Phi(G) / L \cong$ $\Phi(H))$; in particular, if $\Phi(H)=1$ then $\Phi(G)=L$.

PROOF. Let $\left\{b_{i}\right\}_{1 \leq i \leq k}$ be an arbitrary set of generators of $H, G_{i}=\operatorname{gp}\left\{a_{i}\right\}, 1 \leq i \leq k$, an infinite cyclic group (a cyclic group of order $s n_{i}$ if $H$ has no involutions and $s<\infty$ ), where $n_{i}$ is the order of $b_{i}$ in $H, \Omega^{1}$ the free amalgam of the groups $G_{i}$. Then for each $i, 1 \leq i \leq k$, we define a homomorphism $g_{i}: G_{i} \rightarrow H$ by setting $g_{i}\left(a_{i}^{t}\right)=b_{i}^{t}, t \geq 1$. A generating mapping $f$ on $\Omega=\Omega^{1} \backslash\{1\}$ is defined in the following way: if $C \subseteq \Omega$ and $C \nsubseteq G_{i}$ for each $i, 1 \leq i \leq k$, then $f(C)=\Omega$. Hence Theorem A applies to $\Omega^{1}, m=\infty$ (or $m=s$ if $H$ has no involutions) and the mapping $f$ and yields the $k$-generator group $G$ satisfying assertion (1) of the theorem.

By the statement of the theorem, $H$ is a periodic or abelian group. Then it follows from Theorem A and [10, Theorem 33.7] that every non-cyclic subgroup of $G$ has a non-trivial intersection with $L$.

Let $M$ be a non-cyclic subgroup of $G$. Then $M \cap L \neq 1$ and it follows from Theorem A and the definition of the mapping $f$ that $L \leq M$.

It remains to prove that the Frattini subgroup of the group $G$ is isomorphic to an extension of the group $\Phi(H)$ by $L$. It is sufficient to show that every maximal subgroup $M$ of $G$ is an extension of a maximal subgroup of $H$ by the group $L$. But $M$ is not cyclic, for otherwise, $G$ is an extension of a cyclic group by $L$, which contradicts the hypothesis of the theorem. Then by assertion (2) of the theorem, $L \leq M$. The homomorphic image $M_{1}$ of $M$ in $H$ is a maximal subgroup of $H$, since $M$ is a maximal subgroup of $G$; hence $M$ is an extension of $M_{1}$ by $L$. This completes the proof of the theorem.

Another application of Theorem $\mathrm{G}$ was noted by $\mathrm{H}$. Smith and J. Wiegold. It is devoted to the solution of the following problem of J. C. Lennox. Let $\pi$ be an arbitrary 
set of prime numbers, $G$ a finitely generated group such that if $M \leq G$ and $G / M^{G}$ is a finite $\pi$-group, where $M^{G}$ is the normal closure of $M$ in $G$, then $|G: M|$ is a finite $\pi$-number. Lennox asked in [4, problem 8.32] whether the group $G$ is nilpotent and noted that it is true for finitely generated soluble groups. A negative answer to this question follows immediately from

COROLLARY. There is a 2-generator group $G$ having a normal simple infinite subgroup $L$ such that all proper subgroups of $L$ are infinite cyclic, $G / L$ is isomorphic to the free abelian group of rank 2 and if $G / M^{G}$ is a finite group for some subgroup $M$ of $G$, then $M$ is a normal subgroup of $G$.

PROOF. It is sufficient to take $H$ to be the free abelian group of rank 2 and $G$ as the group in Theorem $G$ for $H$ and $s=\infty$. Then if $M \leq G$ is such that $G / M^{G}$ is a finite group, it is easy to see that $M$ is not cyclic, and by assertion (2) of Theorem G, $L \leq M$. Now it is follows from the commutativity of $G / L \cong H$ that $M=M^{G}$.

A subgroup $L$ of a group $G$ is said to be dual-standard if for any subgroups $X, Y$ of $G, \operatorname{gp}\{X, Y\} \cap L=\operatorname{gp}\{X \cap L, Y \cap L\}$. Dual-standard subgroups of finite groups were studied by Zappa [12], those of torsion-free locally soluble groups by Ivanov [1], and Stonehewer and Zacher [11] gave a characterization of dual-standard subgroups of non-periodic locally soluble groups. One more type of dual-standard subgroups is given by the following theorem.

THEOREM H. Let $H$ be an arbitrary non-trivial, at most countable, periodic group, $s$ a sufficiently large odd number or $s=\infty$. Then there exists a group $G$ having a normal dual-standard infinite subgroup $L$ such that $H \cong G / L$ and all proper subgroups of $L$ are infinite cyclic (cyclic groups of order dividing $s$ if $H$ has no involutions and $s<\infty$ ).

PROOF. Let $\left\{b_{i}\right\}_{i \in I}$ be an arbitrary set of generators of $H$. We define groups $G_{i}$, homomorphisms $g_{i}, i \in I$, a set $\Omega$ and a generating mapping $f$ on $\Omega$ as in the proof of Theorem $\mathrm{G}$ (if we consider the set $I$ instead of $\{1, \ldots, k\}$ ). Then Theorem A applies to $\left\{G_{i}\right\}_{i \in l}, m=\infty$ (or $m=s$ if $H$ has no involutions) and the mapping $f$ and yields the group $G$ with the normal infinite subgroup $L$ such that $H \cong G / L$ and all proper subgroups of $L$ are infinite cyclic (cyclic groups of order dividing $s$ if $H$ has no involutions).

By the assumption of the theorem, $H$ is a periodic group; it then follows from Theorem A that every proper subgroup of $G$ has a non-trivial intersection with $L$. Let $A, B$ be arbitrary proper subgroups of $G$. We consider the following cases.

(1) If $\operatorname{gp}\{A, B\}$ is cyclic then it is not hard to show that $\operatorname{gp}\{A, B\} \cap L=\operatorname{gp}\{A \cap$ $L, B \cap L\}$. 
(2) If $\operatorname{gp}\{A, B\}$ is not cyclic then $\operatorname{gp}\{A, B\} \cap L \neq 1$ and it follows from Theorem A and the definition of the mapping $f$ that $L \leq \operatorname{gp}\{A, B\}$. On the other hand, it follows from Theorem A that $\operatorname{gp}\{A \cap L, B \cap L\}$ is not cyclic, and hence $L=\operatorname{gp}\{A \cap L, B \cap L\}$, as required.

In this paper we use the results from [9] and the geometric method of graded diagrams developed by Ol'shanskii (see [10]). Unless otherwise stated, all definitions and notation may be found in [10].

\section{Construction of the group $\mathbf{G}$}

As in [10], we introduce the positive parameters $\alpha, \beta, \gamma, \delta, \varepsilon, \zeta, \eta, \iota$, where all the parameters are arranged according to 'height': that is, the small positive value $\beta$ is chosen after $\alpha, \gamma$ after $\beta$, and so on. Our proofs are based on a system of inequalities involving these parameters. The value of the parameters can be chosen in such a way that all the inequalities hold. We then use the following notation:

$$
\alpha^{\prime}=1 / 2+\alpha, \quad \beta^{\prime}=1-\beta, \quad \gamma^{\prime}=1-\gamma, \quad h=\delta^{-1}, \quad d=\eta^{-1}, n=\iota^{-1} .
$$

We also use the notation introduced at the beginning of Section 1 and fix a sufficiently large odd integer $n_{0}$ such that $n=\left[(h+1)^{-1} n_{0}\right]$, where $[k]$ denotes the integer part of $k$. We set $m=n_{0}$ in the case $m<\infty$.

On the set $W$ we introduce a total order such that $|X| \leq|Y|$ implies $X \leq Y$.

We may assume that $I_{1}$ is a well-ordered set, $t_{1}$ and $t_{2}$ are the minimal and the maximal elements of $I_{1}$, respectively (if such a $t_{2}$ exists), and $\Omega_{1}=\Omega_{2} \cup \Omega_{2}^{-1}$ is the union of two subsets $\Omega_{2}$ and $\Omega_{2}^{-1}$ such that $\Omega_{2} \cap \Omega_{2}^{-1}=\emptyset$ and $\Omega_{2}^{-1}=\left\{a^{-1}, a \in \Omega_{2}\right\}$. We also may assume that $\Omega_{2}$ is a well-ordered set such that if $a \in N_{i}$ and $b \in N_{j}$, where $i<j$, then $a<b$.

By the statement of Theorem A, there is a homomorphism of the free product $G(1)$ of groups $G_{i}, i \in I$, onto $H$ such that its restriction to every group $G_{i}$ is equal to $g_{i}$. Suppose that the kernel of this homomorphism is $N$.

Let $D_{1}=\emptyset$, and suppose, by induction, that we have defined the set of relators $D_{i-1} \subseteq N, i \geq 2$, and set $G(i-1)=\left\langle G(1) \mid R=1 ; R \in D_{i-1}\right\rangle$.

A word $X$ is called free in rank $i-1$ if $X$ is not conjugate in rank $i-1$ to an element of $\Omega^{1}$, that is, to an image in $G(i-1)$ of an element of one of the free factors $G_{j}$. A non-empty word $Y$ is said to be simple in rank $i-1$ if it is free in rank $i-1$, not conjugate in rank $i-1$ (that is, in $G(i-1)$ ) to a power of a shorter word and not conjugate in rank $i-1$ to a power of a period of rank $k<i$.

Now let $P_{i}$ denote a set of words of length $i$ which are simple in rank $i-1$ with the property that $A, B \in P_{i}$ and $A \not \equiv B$ implies that $A$ is not conjugate in rank $i-1$ 
to $B$ or $B^{-1}$. The words in $P_{i}$ are called periods of rank $i$. We may assume (see [9, Lemma 3.1]) that if $a, b \in N_{l} \cap \Omega_{2}, c \in N_{j} \cap \Omega_{2}, d \in N_{s} \cap \Omega_{2}$, where $l<j$ (if such a $j$ exists), $s \neq l$ and $a<b$, then the words $a c, A_{1}=a d b d,[a, c], A_{2}=$ $a[a, c]^{t}, A_{3}=a[a, c]^{-t}, A_{4}=c[a, c]^{-t}, A_{5}=c^{-1}[a, c]^{-t}, A_{6}=\left[(a c)^{k}, c\right], A_{7}=$ $(a c)^{k} A_{6}^{t}, A_{8}=(a c)^{k} A_{6}^{-t}$ are periods of some ranks for each $k, t$, where $100 \zeta^{-1}<$ $k<10^{5} \zeta^{-2}, \zeta n_{0} / 300 \leq t \leq n_{0} / 2$.

For each period $A \in P_{i} \cap N$, we fix a maximal subset $Y_{A}$ such that:

(1) if $T \in Y_{A}$, then $1 \leq|T|<d|A|$;

(2) each double coset of the pair $\operatorname{gp}\{A\}, \operatorname{gp}\{A\}$ of subgroups of $G(i)$ contains at most one word in $Y_{A}$ and this word is of minimal length among the words representing this double coset;

(3) if $T \in Y_{A}$, then $T \in N$ and $F(\{T\}) \subseteq F(\{A\})$.

We may assume (see [9, Lemma 3.1]) that if a period $A$ of some rank is conjugate to a word $\left(B C^{t}\right)^{\varepsilon}$, where $C$ is a period not equal to $[a, c]$ or $A_{6},|\varepsilon|=1, \zeta n_{0} / 300 \leq$ $t \leq n_{0} / 2$ and $B \in Y_{C}$, then $\varepsilon=1$.

For each period $A \in P_{i} \cap N$, we introduce the ordering of the set of natural numbers (or a finite segment of it) on the set $Y_{A}$ such that the first element of the set $Y_{A}$ belongs to $\Omega_{1}$ (it follows from the statement of Theorem A that $Y_{A} \cap \Omega_{1} \neq \emptyset$ ) and if $A=A_{k}, 1 \leq k \leq 8$, or $A=[a, c]$ for some $a, b \in N_{l} \cap \Omega_{2}, c \in N_{j} \cap \Omega_{2}, d \in N_{s} \cap \Omega_{2}$, where $a<b, s \neq l$ and $l<j$, then $a$ is the first element of the set $Y_{A}$. We denote this order by $\leq_{A}$.

The set of relators $S_{i}$ of rank $i$ is constructed as follows. Firstly, if $A \in P_{i}, m<\infty$ and there is a minimal positive integer $k$ such that $A^{k} \in N$, then in the case that $k$ is an odd number, we include in $S_{i}$ a word of the form $A^{k n_{0}}$ (a relator of the first type) and call a relation

$$
A^{k n_{0}}=1
$$

a defining relation of the first type of rank $i$.

For each period $A \in P_{i} \cap N, i \geq 3$, we now construct some relations of the second type. Let $a$ be the minimal element of the set $F(\{A\})$. If $A=A_{j}, j \in\{2,3\}$, for some $a \in N_{l} \cap \Omega_{2}, c \in N_{s} \cap \Omega_{2}$, where $l<s$, then for each $k, 5 \leq k \leq 15$, we introduce the following relations:

$$
c^{-1} A^{n} c A^{n+k} c A^{n+30+k} \cdots c A^{n+30(h-2)+k}=1,
$$

and

$$
a^{-1} A^{n} a A^{n+k} a A^{n+30+k} \cdots a A^{n+30(h-2)+k}=1 .
$$

If $A=A_{j}, j \in\{7,8\}$, for some $a \in N_{l} \cap \Omega_{2}, c \in N_{s} \cap \Omega_{2}$, where $l<s$, then for each $k, t$, where $16 \leq k \leq 25,100 \zeta^{-1}<t<10^{5} \zeta^{-2}$, we consider the relation

$$
a c A^{n}(a c)^{t} A^{n+k}(a c)^{t} A^{n+30+k} \ldots(a c)^{t} A^{n+30(h-2)+k}=1 .
$$


Let $T \in Y_{A}$ and $T \neq a, c$ in the case $A=A_{j}, j \in\{2,3\}$. If $a$ is not contained in $\operatorname{gp}\{A\} \subset G(i-1), T$ is outside $\operatorname{gp}\{A\} a \operatorname{gp}\{A\}$, then we introduce the relation

$$
a A^{n} T A^{n+10} T A^{n+40} \cdots T A^{n+30(h-2)+10}=1,
$$

and if $T$ belongs to $\operatorname{gp}\{A\} a \operatorname{gp}\{A\}$, then it follows from [10, Lemma 25.18] that $T$ is not contained in $\operatorname{gp}\{A\} a^{-1} \operatorname{gp}\{A\}$ in $G(i-1)$, and we set

$$
a^{-1} A^{n} T A^{n+10} T A^{n+40} \ldots T A^{n+30(h-2)+10}=1 .
$$

If $T \in Y_{A}$ and $T \neq(a c)^{t}, 100 \zeta^{-1}<t<10^{5} \zeta^{-2}$, in the case $A=A_{j}, j \in\{7,8\}$, then we introduce the relation

$$
a^{-1} A^{n} T A^{n+20} T A^{n+50} \cdots T A^{n+30(h-2)+20}=1 .
$$

And if $T \in Y_{A}$ then let $T_{1}$ be the minimal element of the set $Y_{A}$ such that $T_{1}$ is not contained in neither $\operatorname{gp}\{A\} \subset G(i-1)$ nor in $\operatorname{gp}\{A\} a^{ \pm 1} \operatorname{gp}\{A\}$ and $T<_{A} T_{1}$ (if such an element $T_{1}$ exists). Then we consider the relation

$$
T_{1} A^{n} T A^{n+30} T A^{n+60} \cdots T A^{n+30(h-1)}=1 .
$$

Relations (2.2)-(2.8) are called defining relations of the second type of rank $i$, and their left-hand sides are called relators of the second type of rank $i$, and are included in $S_{i}$. For each $i \geq 2$, we set $D_{i}=D_{i-1} \cup S_{i}$, and the group $G(i)$ is defined by its presentation:

$$
G(i)=\left\langle G(1) \mid R=1 ; R \in D_{i}\right\rangle .
$$

Finally, we define $G=\left\langle G(1) \mid R=1 ; R \in D=\bigcup_{i \geq 1} D_{i}\right\rangle$.

By a diagram of rank $i$, where $i \geq 2$, we mean a diagram over the presentation (2.9). Relators of the first type (in the case $m<\infty$ ) correspond, in the diagrams under considerations, to cells of the first type whose contour (that is, boundary path) is taken as one long cyclic section. But if a cell $\Pi$ corresponds to a word of the form (2.2)-(2.8), then it is called a cell of the second type. Its contour splits into sections according to (2.2)-(2.8). Those sections of $\Pi$ with labels $\left(A^{n+s}\right)^{ \pm 1}$ are called long sections while the others (with labels $T^{ \pm 1}, a^{ \pm 1},(a c)^{ \pm 1}$ and $T_{1}^{ \pm 1}$ ) are called short sections of the contour.

\section{Auxiliary lemmas}

Immediate verification shows that the above presentations of the groups $G(i)$ satisfy condition $R$ (see [10, $\S 25,34]$ ). So we can apply to diagrams over the presentation (2.9) all the results in [10, Chapter 11]. 
LEMMA 1. Let $X=Y$ in $G$, where $Y$ is a minimal word of the group $G$. Then $F(\{Y\}) \subseteq F(\{X\})$.

PROOF. Let $\Delta$ be a reduced circular diagram of some rank with contour $p_{1} p_{2}$, where $\phi\left(p_{1}\right) \equiv X^{-1}, \phi\left(p_{2}\right) \equiv Y$. If $r(\Delta)=0$, then we derive the conclusion of the lemma from the definition of the mapping $F$.

If $r(\Delta)>0$, then by [10, Theorem 22.1], there is a $\gamma$-cell $\pi$ in $\Delta$. We proceed by induction on $|\Delta(2)|$. It follows from [10, Lemma 21.7] that there is a contiguity submap $\Gamma$ of $\pi$ to $p_{1}$ such that $\left(\pi, \Gamma, p_{1}\right)>\varepsilon$, since $\gamma^{\prime}-\alpha^{\prime}>\varepsilon$. Repeating the proof of [10, Theorem 22.2], we obtain that there is a long section $p$ of a $D$-cell $\Pi$ in $\Delta$ and a contiguity submap $\Gamma_{1}$ of $p$ to $p_{1}$ such that $r\left(\Gamma_{1}\right)=0$ and $\left(p, \Gamma_{1}, p_{1}\right) \geq \varepsilon$. By the definition of the relations of $G$, if $\Pi$ is a cell of the second type and $t_{1}, t_{2}$ are its short and long sections, respectively, then $F\left(\left\{\phi\left(t_{1}\right)\right\}\right) \subseteq F\left(\left\{\phi\left(t_{2}\right)\right\}\right)$. Therefore, excising $\Pi$ from $\Delta$ together with $\Gamma_{1}$, we obtain a diagram $\Delta_{1}$ of an equation $X_{1}^{-1} Y=1$ with $\left|\Delta_{1}(2)\right|<|\Delta(2)|$, and $F\left(\left\{X_{1}\right\}\right) \subseteq F(\{X\})$. By the induction hypothesis we can assume the lemma is true for this equation. Hence $F(\{Y\}) \subseteq F\left(\left\{X_{1}\right\}\right) \subseteq F(\{X\})$, as required.

LEMMA 2. Let $\Gamma$ be a contiguity submap of $q_{1}^{\prime}$ to $q_{2}^{\prime}$ in a $B$-diagram $\Delta$ and $\phi\left(q_{1}^{\prime}\right), \phi\left(q_{2}^{\prime}\right)$ minimal words in $G$, where $q_{1}^{\prime}$ and $q_{2}^{\prime}$ are sections of cells or of contours of $\Delta$. If $\partial\left(q_{1}^{\prime}, \Gamma, q_{2}^{\prime}\right)=p_{1} q_{1} p_{2} q_{2}$, then the following conditions hold:

(1) $F\left(\left\{\phi\left(p_{i}\right)\right\}\right) \subseteq F\left(\left\{\phi\left(q_{j}\right)\right\}\right)$ for each $i, j \in\{1,2\}$;

(2) $\quad F\left(\left\{\phi\left(q_{1}\right)\right\}\right)=F\left(\left\{\phi\left(q_{2}\right)\right\}\right)$.

ProOF. We denote by $E_{1}$ and $E_{2}$ the bonds defining $\Gamma$. If $E_{1}$ and $E_{2}$ are 0 -bonds, then $\left|p_{1}\right|=\left|p_{2}\right|=0$, and we derive the conclusion of the lemma from Lemma 1 .

Let $\pi$ be the principal cell of $E_{1}$ and $r(\pi)=k>0$. By definition of the bond, there are contiguity submaps $\Gamma_{1}, \Gamma_{2}$ of long sections $t_{1}$ and $t_{2}$ of $\pi$ to $q_{1}^{\prime}$ and $q_{2}^{\prime}$ such that $\left(t_{i}, \Gamma_{i}, q_{i}^{\prime}\right) \geq \varepsilon, i=1,2$. We denote by $p_{1}^{i} q_{1}^{i} p_{2}^{i} q_{2}^{i}$ the standard decomposition of the contour $\partial \Gamma_{i}$, where $\Gamma_{i} \wedge q_{i}^{\prime}=q_{2}^{i}, \Gamma_{i} \wedge t_{i}=q_{1}^{i}, i=1,2$. Since $\Gamma_{1}$ and $\Gamma_{2}$ have fewer $D$-cells than $\Delta$, then by the induction hypothesis,

$$
F\left(\left\{\phi\left(q_{1}^{i}\right)\right\}\right)=F\left(\left\{\phi\left(q_{2}^{i}\right)\right\}\right), \quad i=1,2,
$$

and

$$
F\left(\left\{\phi\left(p_{j}^{i}\right)\right\}\right) \subseteq F\left(\left\{\phi\left(q_{2}^{i}\right)\right\}\right)
$$

for each $i, j \in\{1,2\}$. It follows from the definition of the mapping $F$ and the relations of $G$ that

$$
F(\{\phi(\partial \pi)\})=F\left(\left\{\phi\left(q_{1}^{i}\right)\right\}\right)
$$


for each $i \in\{1,2\}$.

The path $p_{1}$ has the form $p_{1}^{2} u p_{2}^{1}$, where $u^{-1}$ is a subpath in $\partial \pi$. Then by the definition of the mapping $F$,

$$
F(\{\phi(u)\}) \subseteq F(\{\phi(\partial \pi)\})
$$

It follows from (3.1)-(3.4) that

$$
F\left(\left\{\phi\left(p_{1}\right)\right\}\right) \subseteq F\left(\left\{\phi\left(p_{1}^{2}\right), \phi(u), \phi\left(p_{2}^{1}\right)\right\}\right) \subseteq F\left(\left\{\phi\left(q_{2}^{i}\right)\right\}\right)
$$

for each $i \in\{1,2\}$. Hence $F\left(\left\{\phi\left(p_{1}\right)\right\}\right) \subseteq F\left(\left\{\phi\left(q_{i}\right)\right\}\right), i=1,2$.

Similarly we obtain the required assertion for $F\left(\left\{\phi\left(p_{2}\right)\right\}\right)$. Now it follows from Lemma 1 that

$$
F\left(\left\{\phi\left(q_{i}\right)\right\}\right) \subseteq F\left(\left\{\phi\left(q_{3-i}\right), \phi\left(p_{1}\right), \phi\left(p_{2}\right)\right\}\right) \subseteq F\left(\left\{\phi\left(q_{3-i}\right)\right\}\right)
$$

for each $i \in\{1,2\}$. This completes the proof of the lemma.

LEMMA 3. Let $V$ be a minimal word in $G$ and $V=Z^{-1} A^{t} Z$, where $A$ is a period of some rank, $Z$ is a minimal word in $G$, or $V=Z^{-1} a_{j} Z$, where $a_{j} \in G_{i}$ for some $i \in I$ and $Z$ is of minimal length among the words representing a coset $G_{i} Z$. Then $F(\{V\})=F(\{Z, A\})\left(F(\{V\})=F\left(\left\{Z, a_{j}\right\}\right)\right)$.

Proof. Consider, for example, the first case (the other case of the lemma can be considered in the same manner).

By Lemma $1, F(\{V\}) \subseteq F(\{Z, A\})$ : hence it is necessary to show the reverse inclusion. We note that for this purpose it is sufficient to find $X \in G$ such that $V=$ $X^{-1} A^{t} X$ and $F(\{V\}) \supseteq F(\{X, A\})$, since by [10, Lemma 34.9] $Z X^{-1} \in \operatorname{gp}\{A\}$, and it follows from Lemma 1 that $F(\{Z\}) \subseteq F(\{X, A\})$, hence $F(\{Z, A\}) \subseteq F(\{X, A\}) \subseteq$ $F(\{V\})$.

Let $V=Y^{-1} V_{1} Y$ in the group $G(1)$, where $V_{1}$ is cyclically minimal in $G(1)$. Then there is a reduced annular diagram $\Delta$ of some rank with contours $p$ and $q$, where $\phi(p) \equiv V_{1}$ and $\phi(q) \equiv A^{-t}$.

Repeating the proof of Lemma 1, we obtain that for each cell $\pi$ in $\Delta, F(\{\phi(\partial \pi)\}) \subseteq$ $F\left(\left\{V_{1}\right\}\right)$ and

$$
F(\{A\}) \subseteq F\left(\left\{V_{1}\right\}\right)
$$

Therefore, there exists a word $L$ such that $V_{1}=L^{-1} A^{t} L$ and

$$
F(\{L\}) \subseteq F\left(\left\{V_{1}, A\right\}\right) \subseteq F\left(\left\{V_{1}\right\}\right)
$$


We have that $V=(L Y)^{-1} A^{t}(L Y)=X^{-1} A^{l} X$, and by Lemma 1 and (3.5), (3.6),

$$
F(\{X, A\}) \subseteq F\left(\left\{V_{1}, L, Y\right\}\right) \subseteq F(\{V\}) .
$$

The proof of the lemma is complete.

A reduced diagram $\Delta$ of rank $i$ on a sphere with three holes with contours $q_{1}^{0}, q_{2}^{0}, q_{3}^{0}$ is called $I$-diagram if the following conditions hold:

I1. sections $q_{1}^{0}$ and $q_{2}^{0}$ have labels $A^{k}$ and $A^{-k}$, respectively, where $A$ is either a simple word in rank $i$ or a period of rank $j \leq i, 100 \zeta^{-1}<k$ (and $k \leq n_{0} / 2$ if $A$ is a period of the first type);

12. the section $q_{3}^{0}$ is cyclically reduced;

13. if $\Gamma$ is a contiguity submap of $q_{i_{1}}^{0}$ to $q_{i_{2}}^{0}$, where $i_{1}, i_{2} \in\{1,2\}$ and $i_{1} \neq i_{2}$, then $\left(q_{i_{1}}^{0}, \Gamma, q_{i_{2}}^{0}\right)<1 / 100$ and $\left(q_{i_{2}}^{0}, \Gamma, q_{i_{1}}^{0}\right)<1 / 100$;

I4. if $\Gamma$ is a contiguity submap of a long section $p$ of a cell $\Pi$ to $q_{3}^{0}$, then $\left(p, \Gamma, q_{3}^{0}\right)<$ $\varepsilon$.

LEMMA 4. In any I-diagram $\Delta$, there are contiguity submaps $\Gamma_{1}$ and $\Gamma_{2}$ of $q_{1}^{0}$ to $q_{3}^{0}$ and $q_{2}^{0}$ to $q_{3}^{0}$, respectively, such that $r\left(\Gamma_{i}\right)=0$ and $\left(q_{i}^{0}, \Gamma_{i}, q_{3}^{0}\right)>1 / 10, i=1,2$.

PROOF. We consider the following cases.

(1) Let $s$ be a section of a cell $\pi$ or a subpath of a section $q_{i}^{0}, i=1,2$, and $\Gamma$ a contiguity submap of $s$ to $q_{3}^{0}$. Then by condition I4, $\Gamma$ is the 0 -contiguity submap with contour $p_{1} s_{1} p_{2} s_{2}$, where $\left|p_{1}\right|=\left|p_{2}\right|=0$ and $s_{1}, s_{2}$ are subpaths of sections $s$ and $q_{3}^{0}$, respectively. If $r(\Gamma)>0$ then by [10, Theorem 22.1], there is a $\gamma$-cell $\Pi$ in $\Gamma$. It follows from [10, Lemma 21.7] that for any contiguity submap $\Gamma_{1}$ of $\Pi$ to $s_{1}$, the $\Gamma_{1}$-contiguity degree of $\Pi$ to $s_{1}$ is less than $\alpha^{\prime}$; hence there exists a contiguity submap $\Gamma_{2}$ of $\Pi$ to $q_{3}^{0}$ such that $\left(\Pi, \Gamma_{2}, q_{3}^{0}\right)>\varepsilon$, and we arrive at a contradiction to condition I4. Thus $r(\Gamma)=0$.

(2) Let $\Gamma$ be a contiguity submap of $q_{3}^{0}$ to $q_{3}^{0}$. Then by condition I4 and [10, Theorem 22.1], we obtain, as in case 1 , that $r(\Gamma)=0$, since $2 \varepsilon<\gamma^{\prime}$.

(3) We define the distinguished contiguity submaps in an I-diagram in the same way as for E-maps. The $\Omega$-edges of the contiguity arcs of $q_{i}^{0}$ to $q_{i^{\prime}}^{0}$, where $i \in\{1,2\}, i^{\prime} \in$ $\{1,2,3\}$, for the distinguished submaps are called outer edges in $\Delta$ while all the other edges are called inner. The construction of the estimating graphs and the weight function is left unchanged. We obtain estimates for the sums $H^{\prime}, C^{\prime}, D^{\prime}$ and $G^{\prime}$ in the same way as in [10, Lemma 24.6].

Let $K^{\prime}$ be defined for an I-diagram in the same way as in [10, Lemma 23.8] for a C-map. If $q_{2}^{\prime}=q_{3}^{0}$ then by case 1 and condition I4, $\left|q_{2}\right|=\left|q_{1}\right|<\varepsilon\left|q_{1}^{\prime}\right|$ (notation from [10, Lemma 23.8]). Then, as in [10, Lemma 23.8], we obtain $K^{\prime} \leq 10 \varepsilon^{2 / 3} M$. 
Now $L^{\prime}$ can be defined as the sum $L$ in [10, Lemma 23.12] (sections of the contour of the first kind are now replaced by $q_{1}^{0}, q_{2}^{0}$ and $q_{3}^{0}$ ). If $q=q_{3}^{0}$ then by case 1 , $\left|q_{2}\right|=\left|q_{1}\right|<d k$ (notation from [10, Lemma 23.12]). As in that lemma, we have $L^{\prime} \leq \alpha M$. Then as in [10, Lemma 24.6], immediate verification shows that

$$
M<\alpha \nu(\Delta) .
$$

(4) Let $\Gamma$ be a contiguity submap of $q_{3}^{0}$ to $q_{3}^{0}$ and $\partial\left(q_{3}^{0}, \Gamma, q_{3}^{0}\right)=p_{1} s_{1} p_{2} s_{2}$. Then by case $2, r(\Gamma)=0$, and it follows from condition $\mathrm{I} 2$ that $\Delta$ consists of two annular subdiagrams $\Delta_{1}$ and $\Delta_{2}$ with contours $t_{1} q_{1}$ and $t_{2} q_{2}$, respectively, where $t_{1}, t_{2}$ are subpaths of $q_{3}^{0}$, such that $\Delta_{1}$ and $\Delta_{2}$ are joined in $\Delta$ by subpaths $s_{1}, s_{2}$ of $q_{3}^{0}$. Applying condition I4, [10, Theorem 22.1 and Lemma 21.7] to $\Delta_{i}, i=1,2$, we obtain, as in case 1, that $r\left(\Delta_{i}\right)=0$, which completes the proof of the lemma in this case.

(5) It remains to consider the case when $\Delta$ has no contiguity submaps of $q_{3}^{0}$ to $q_{3}^{0}$. It follows from [10, Lemma 25.8] that there is no contiguity submap $\Gamma_{i}$ of $q_{i}^{0}$ to $q_{i}^{0}, i=1,2$, such that $\left(q_{i}^{0}, \Gamma_{i}, q_{i}^{0}\right)>1 / 100$. Then by (3.7) and condition $\mathrm{I} 3$, there are distinguished contiguity submaps $\Gamma_{1}, \Gamma_{2}$ of $q_{1}^{0}, q_{2}^{0}$ to $q_{3}^{0}$, respectively, such that the sum of the weights of the contiguity arcs $s_{1}=\Gamma_{1} \wedge q_{1}^{0}$ and $s_{2}=\Gamma_{2} \wedge q_{2}^{0}$ is greater than

$$
(1-\alpha-4 / 100) v(\Delta)>9 v(\Delta) / 10 .
$$

But by condition I1 and the definition of the weight function,

$$
v\left(q_{1}^{0}\right)=v\left(q_{2}^{0}\right)=v(\Delta) / 2 .
$$

It follows from (3.8) and (3.9) that $\Gamma_{i}$ exists for each $i \in\{1,2\}$, and in the light of case 1 , we have the conclusion of the lemma.

LEMMA 5. Let $A$ and $C$ be periods of the group $G, V \equiv C^{k}$, where $100 \zeta^{-1}<k$ (and $k \leq n_{0} / 2$ if $C$ is a period of the first type), $W$ a word which does not commute with $V$ in $G$ and whose length is minimal among all words in the double coset $\operatorname{gp}\left\{C^{k}\right\} W \operatorname{gp}\left\{C^{k}\right\}$, and also let $C^{k} W C^{-k} W^{-1}=Z^{-1} A^{l} Z$, where $Z$ is a minimal word in $G$ (and $|l| \leq n_{0} / 2$ if $A$ is a period of the first type). Then $|l| \leq 100 \zeta^{-1}$ and, by a simultaneous conjugation in $G$, we can bring $\left(\left[C^{k}, W\right], C^{k}\right)$ to the form $\left(A^{l}, B\right)$, where $B$ is a minimal word in $G,|B|<d|A|$ and

$$
F(\{A\})=F(\{C, W\}), \quad F(\{B\}) \subseteq F(\{A\}) .
$$

PROOF. By [10, Lemma 25.21], it remains to prove only (3.10). It follows from Lemmas 1 and 3 that $F(\{A, Z\}) \subseteq F(\{C, W\})$; hence

$$
F(\{A\}) \subseteq F(\{C, W\}) .
$$


Now let $\Delta$ be a reduced annular diagram (of some rank) with contours $p$ and $q$ such that $\phi(q) \equiv A^{-l}, p=p_{1} p_{2} p_{3} p_{4}, \phi\left(p_{1}\right) \equiv \phi\left(p_{3}^{-1}\right) \equiv C^{k}, \phi\left(p_{2}\right) \equiv \phi\left(p_{4}^{-1}\right) \equiv W$. Pasting together paths $p_{2}$ and $p_{4}^{-1}$, we obtain a diagram $\Delta^{\prime}$ on a sphere with three holes whose reduced form (that is, with $j$-pairs removed) is denoted by $\Delta_{0}$. The cyclic sections $p_{1}, p_{3}$ and $q$ can be assumed smooth in $\Delta_{0}$ if we modify their labels in accordance with [10, Lemma 13.3].

It is obvious that $\Delta_{0}$ satisfies conditions I1 and I2. Suppose that there is a contiguity submap $\Gamma$ of $p_{i_{1}}$ to $p_{i_{2}}$, where $i_{1}, i_{2} \in\{1,3\}$ and $i_{1} \neq i_{2}$, such that $\left(p_{i_{1}}, \Gamma, p_{i_{2}}\right) \geq$ $1 / 100$. We have that $|C|=\left|C^{-1}\right|$; then by [10, Lemma 25.10], $p_{1}$ and $p_{3}$ are $C$ compatible in $\Delta_{0}$, and using [10, Lemma 24.9], we arrive at a contradiction to the choice of the word $W$. Thus $\Delta_{0}$ satisfies condition I3.

Now we assume that there is a long section $t$ of a $D$-cell $\pi$ in $\Delta_{0}$ and a contiguity submap $\Gamma$ of $t$ to $q$ such that $(t, \Gamma, q) \geq \varepsilon$. Then repeating the proof of [10, Theorem 22.2], we obtain that there is a cell $\pi_{1}$ and a contiguity submap $\Gamma_{1}$ of a long section $t_{1}$ of $\pi_{1}$ to $q$ such that $r\left(\Gamma_{1}\right)=0$ and $\left(t_{1}, \Gamma_{1}, q\right) \geq \varepsilon$. Excising the cell $\pi_{1}$ together with $\Gamma_{1}$ from $\Delta_{0}$, we obtain a diagram $\Delta_{1}$ on a sphere with three holes with cyclic sections $p_{1}, p_{3}$ and $q_{1}$ such that $\left|\Delta_{1}(2)\right|<|\Delta(2)|$. We can assume that the section $q_{1}$ is cyclically reduced, and by the definition of the relations of $G, F\left(\left\{\phi\left(q_{1}\right)\right\}\right) \subseteq$ $F(\{\phi(q)\})$. Then, by repeating the same trick several times, we obtain an I-diagram $\Delta_{r}$ with cyclic sections $p_{1}, p_{3}$ and $q_{r}$ such that

$$
F(\{C\}) \subseteq F\left(\left\{\phi\left(q_{r}\right)\right\}\right) \subseteq F(\{\phi(q)\})=F(\{A\}) .
$$

Moreover, the initial points of $p_{1}$ and $p_{3}$ can be joined in $\Delta_{r}$ by a path $s$ of the form $s_{1} s^{\prime} s_{3}$, where $s^{\prime}, s_{1}$ and $s_{3}$ are subpaths of $q_{r}, p_{1}$ and $p_{3}$, respectively. Then by [10, Lemma 24.9], a word $\phi(s)$ is contained in $\operatorname{gp}\left\{C^{k}\right\} W \operatorname{gp}\left\{C^{k}\right\}$, and it follows from the choice of the word $W$, Lemma 1 and (3.12) that

$$
F(\{W\}) \subseteq F(\{A\}) .
$$

It follows from (3.11)-(3.13) that $F(\{C, W\})=F(\{A\})$, and by Lemmas 1 and 3 that $F(\{Z, A\}) \subseteq F(\{C, W\})=F(\{A\})$. Hence

$$
F(\{Z\}) \subseteq F(\{A\}) .
$$

But the word $B$ is minimal in $G$ and equal in $G$ to the word $Z C^{k} Z^{-1}$. Then by Lemma 3, (3.12) and (3.14), $F(\{B\})=F(\{Z, C\}) \subseteq F(\{A\})$, which completes the proof of the lemma.

Lemma 6. Let $R=\operatorname{gp}\left\{C^{k}, W\right\}$, where $C$ is a period, $C^{k} \in N \backslash\{1\}$ and $W$ is a minimal word in $G$ such that $W$ is not contained in $\mathrm{gp}\{C\}$. Then $R$ contains a period $C_{1} \in N$ such that $F\left(\left\{C_{1}\right\}\right)=F(\{C, W\})$ and $n|C|<\left|C_{1}\right|$. 
Proof. We can assume that $C^{t} \in R \cap N$, where $n_{0} / 5<t$ (and $t \leq n_{0} / 2$ if $C$ is a period of the first type). By [10, Lemma 34.9], $\left[C^{t}, W\right] \neq 1$. It follows from Lemma 1 that we can assume $W$ has minimal length among all words in the double coset $\operatorname{gp}\left\{C^{t}\right\} W \operatorname{gp}\left\{C^{t}\right\}$, and by Lemma 5 and [10, Lemma 34.7], [C,$\left.W\right]=Z^{-1} A^{f} Z$, where $A$ is a period, $Z$ is a minimal word in $G,|f| \leq 100 \zeta^{-1},|B|<d|A|$ for a word $B$ which is minimal in $G$ and equal in $G$ to the word $Z C^{t} Z^{-1}$, and condition (3.10) holds. Moreover, it follows from the proof of [10, Lemma 25.21] that

$$
|A|>10^{-2} \zeta^{2}\left|C^{t}\right|>\zeta^{2} n_{0}|C| / 600
$$

and

$$
|Z|<400 \zeta^{-2}|A| \text {. }
$$

Raising $A^{f}$ to a suitable power we consider the subgroup $\operatorname{gp}\left\{B, A^{p}\right\}$ of the group $R_{1}=Z R Z^{-1}$, where $n_{0} / 3 \leq p \leq 2 n_{0} / 3$. Repeating the proof of [10, Lemma 27.3], we obtain that $B A^{p}=Z_{1}^{-1} C_{1}^{\varepsilon} Z_{1}$, where $|\varepsilon|=1, C_{1}$ is a period of some rank such that $C_{1} \in N, Z_{1}$ is a minimal word in $G$ and

$$
\left|Z_{1}\right|<2\left|C_{1}\right|, \quad n_{0}|A| / 100<\left|C_{1}\right| .
$$

Now let $\Delta$ denote a reduced annular diagram for this conjugacy. Let $z l$ and $q$ be the contours of $\Delta$, where $\phi(z) \equiv B, \phi(l) \equiv A^{p}, \phi\left(q^{-1}\right) \equiv C_{1}^{\varepsilon}$. Then, as in the proof of [10, Lemma 27.3], there is a contiguity submap $\Gamma$ of $l$ to $q$ in $\Delta$ such that $(l, \Gamma, q)>\beta^{\prime}$. Hence by Lemma $2, F(\{A\}) \subseteq F\left(\left\{C_{1}\right\}\right)$. But it follows from Lemma 3 and (3.10) that

$$
F\left(\left\{Z_{1}, C_{1}\right\}\right) \subseteq F(\{B, A\}) \subseteq F(\{A\})
$$

Thus

$$
F\left(\left\{C_{1}\right\}\right)=F(\{A\}), F\left(\left\{Z_{1}\right\}\right) \subseteq F(\{A\}) .
$$

We consider the subgroup $\operatorname{gp}\left\{C_{1}, Z_{2}\right\}$ of the group $R_{2}=Z_{1} R_{1} Z_{1}^{-1}=$ $\left(Z_{1} Z\right) R\left(Z_{1} Z\right)^{-1}$, where $Z_{2}$ is a minimal word in $G$ which is equal in $G$ to the word $Z_{1} B Z_{1}^{-1}$. It follows from the proof of [10, Lemma 27.3] that $\left|Z_{2}\right|<3\left|C_{1}\right|$, and by Lemma 1 and (3.10), (3.18),

$$
F\left(\left\{Z_{2}\right\}\right) \subseteq F\left(\left\{Z_{1}, B\right\}\right) \subseteq F\left(\left\{C_{1}\right\}\right) .
$$

It follows from Lemma 1, (3.10) and (3.16)-(3.19) that there are $Z_{i}^{\prime} \in Y_{C_{1}}, i \in$ $\{1,2\}$, and $Z^{\prime} \in Y_{C_{1}}$ such that $Z_{i} \in \operatorname{gp}\left\{C_{1}\right\} Z_{i}^{\prime} \operatorname{gp}\left\{C_{1}\right\}, i \in\{1,2\}$, and $Z \in \operatorname{gp}\left\{C_{1}\right\} Z^{\prime}$ $\mathrm{gp}\left\{C_{1}\right\}$. By the definition of the relation (2.5) for $C_{1}$ and $Z_{2}^{\prime}$, the minimal element $a$ 
of the set $Y_{C_{1}}$ is contained in $R_{2}$. Now using the defining relation (2.8) for $C_{1}$ and $a$, we obtain that $a_{1} \in R_{2}$, where $a_{1}$ is the minimal element of the set $Y_{C_{1}} \backslash\{a\}$, and so on. Thus we have that $Z^{\prime}$ and $Z_{1}^{\prime}$ are contained in $R_{2}$; hence $Z, Z_{1} \in R_{2}$ and $R=R_{2}$.

Finally, it follows from (3.15) and (3.17) that $n|C|<\left|C_{1}\right|$, which completes the proof of the lemma.

\section{Proof of Theorem A}

Let $L$ be the homomorphic image of the group $N$ in $G$. Then $L$ is a normal subgroup of $G$, and it follows from the definition of the relations of $G$ made in Section 2 that $G / L \cong F / N \cong H$. By [10, Lemma 34.13], a group $\operatorname{gp}\left\{\Omega_{1}\right\}$ is infinite; hence $L$ is an infinite subgroup of $G$. It follows from [10, Lemma 25.1] that the group $G$ is aspherical and atoroidal.

If $X \in L$ and $X$ is not conjugate in $G$ to an element of any $G_{i}, i \in I$, then, by [10, Lemma 34.7], $X$ is conjugate to a power of a period $Y$, and it follows from the definition of the relation (2.1) that either $X$ is of order dividing $m$ (of infinite order in the case $m=\infty$ ) or the homomorphic image of $Y$ in $H$ has even order and $Y$ is of infinite order.

Repeating the proof of $[9$, Theorem A], we obtain that every automorphism of $L$ is induced by an inner automorphism of $G$; hence Aut $L \cong G$ and Out $L \cong H$. The claim about regular automorphisms of $L$ follows from [10, Lemmas 34.9 and 34.11].

Let $M$ be an arbitrary non-cyclic subgroup of $G$. If $M$ has no free elements, then by the proof of [10, Theorem 35.1], $M$ is conjugate to a subgroup $M_{1}$ of a group $G_{i}, i \in I$, and so $M$ is conjugate to a subgroup $G_{C, M_{1}^{\prime}}$, where $C=\left(M_{1} \cap L\right) \backslash\{1\}$ and $M_{1}^{\prime}$ is the homomorphic image of $M_{1}$ in $H$.

Let $M$ contain a free element $X$ of $G$. By [10, Lemma 34.7], $X$ is conjugate to a power of a period $A$. If $M \cap L=1$, then it follows from the definition of the relation (2.1) that the image $A$ in $H$ has infinite order. In the opposite case, the group $M$ is conjugate to a subgroup $M_{1}$ containing $A^{k}$ and $W$, where $100 \zeta^{-1}<k$ (and $k \leq n_{0} / 2$ if $A$ is a period of the first type), $W$ is a word which does not commute with $A^{k}$ in $G$ and whose length is minimal among all words in the double coset $\operatorname{gp}\left\{A^{k}\right\} W \operatorname{gp}\left\{A^{k}\right\}$, and moreover, $\left[A^{k}, W\right]$ is contained in $L$. It follows from Lemma 5 that $M_{1}$ is conjugate in $G$ to a subgroup $M_{2}=\operatorname{gp}\left\{C^{l},\left\{W_{j}\right\}_{j \in J}\right\}$, where $C$ is a period, $C^{l} \in L$ and for each $j \in J, W_{j}$ is a minimal word in $G$ such that $W_{j}$ is not contained in $\operatorname{gp}\{C\}$.

Of course, $M_{2}$ is an extension of a group $H^{\prime}$ by a normal subgroup $L^{\prime}=M_{2} \cap L$, where $H^{\prime}$ is the homomorphic image of $M_{2}$ in $H$. Let $K=F\left(\{C\} \cup\left\{W_{j}\right\}_{j \in J}\right)$. By Lemma $1, M_{2} \leq R_{K}$ and $L^{\prime} \leq L_{K}=R_{K} \cap L$.

Now we prove that $L_{K} \leq L^{\prime}$. Let $X$ be an arbitrary element of $L_{K}$. Then by the definition of a generating mapping on $\Omega$, there are $W_{i_{1}}, \ldots, W_{i_{t}}, t \geq 1$, such 
that $F(\{X\}) \subseteq F\left(\left\{C, W_{i_{1}}, \ldots, W_{i_{1}}\right\}\right)$. Applying Lemma 6 to the group $\operatorname{gp}\left\{C^{l}, W_{i_{1}}\right\}$, we obtain that the group $L^{\prime}$ contains a period $C_{1}$ such that $F\left(\left\{C_{1}\right\}\right)=F\left(\left\{C, W_{i_{1}}\right\}\right)$. Similarly, $\operatorname{gp}\left\{C_{1}, W_{i_{2}}\right\}$ contains a period $C_{2} \in L^{\prime}$ such that $F\left(\left\{C_{2}\right\}=F\left(\left\{C, W_{i_{1}}, W_{i_{2}}\right\}\right)\right.$, and so on. As a result, we have a period $C_{t} \in L^{\prime}$ such that $F(\{X\}) \subseteq F\left(\left\{C_{t}\right\}\right)$. If $|X|>d\left|C_{t}\right|$, then by Lemma 6 , the subgroup $\operatorname{gp}\left\{C_{t}, C^{\prime}\right\}$ contains a period $C_{t+1}$ such that $F(\{X\}) \subseteq F\left(\left\{C_{t+1}\right\}\right)$ and $n\left|C_{t}\right|<\left|C_{t+1}\right|$. Repeating the same trick several times, we have that $L^{\prime}$ contains a period $B$ such that $F(\{X\}) \subseteq F(\{B\})$ and $|X|<d|B|$. We may assume that $C^{l} \in Y_{B}$, and it follows from the definition of the relation (2.5) for $B$ and $C^{l}$ that $a \in L^{\prime}$, where $a$ is the minimal element of the set $Y_{B}$. Now using the defining relation (2.8) for $B$ and $a$, we obtain that $a_{1} \in L^{\prime}$, where $a_{1}$ is the minimal element of the set $Y_{B} \backslash\{a\}$, and so on. As a result, we have that $X_{1} \in L^{\prime}$, where $X_{1} \in Y_{B}$ such that $X$ is contained in $\operatorname{gp}\{B\} X_{1} \operatorname{gp}\{B\}$. Then $X \in L^{\prime}$ and $L_{K} \leq L^{\prime}$.

If $C \nsubseteq G_{i}$ for each $i \in I$, then by the statement of Theorem A, $f(C) \cap \Omega_{1} \neq \emptyset$. Let $a \in f(C) \cap \Omega_{1}$ and $L_{C}^{\prime}=\operatorname{gp}\left\{b a b^{-1}, b \in f(C)\right\}$. It is obvious that $L_{C}^{\prime} \leq L_{C}$. Now we prove that $L_{C} \leq L_{C}^{\prime}$. We have that $C \nsubseteq G_{i}$ for each $i \in I$; then, by [10, Lemma 34.11] and the definition of the relations of $G$, there is $b \in f(C)$ and $\varepsilon,|\varepsilon|=1$, such that $[a, b]^{\varepsilon}$ is a period. Let $X$ be an arbitrary element of $L_{C}$. Then by the definition of a generating mapping on $\Omega$, there are $b_{1}, \ldots, b_{t}, t \geq 1$, such that $F(\{X\}) \subseteq F\left(\left\{[a, b], b_{1} a b_{1}^{-1}, \ldots, b_{t} a b_{t}^{-1}\right\}\right)$. Repeating the previous considerations for $X$ and the set $\left\{[a, b], b_{1} a b_{1}^{-1}, \ldots, b_{t} a b_{t}^{-1}\right\}$, we obtain that $X \in L_{C}^{\prime}$. Then $L_{C} \leq L_{C}^{\prime}$, as required.

Assertion 7 of Theorem A follows from Lemma 1.

Let $C \nsubseteq G_{i}$ for each $i \in I, M$ be a subgroup of $G$ in which every element is a minimal word of $G, L_{C_{1}}^{\prime}=\operatorname{gp}\left\{L_{C}, M\right\} \cap L$ and $C_{1}=F(C \cup(M \backslash\{1\}))$. It follows from Lemma 3 that $L_{C_{1}}^{\prime} \leq L_{C_{1}}$. Now we prove that $L_{C_{1}} \leq L_{C_{1}}^{\prime}$. We have that $C \nsubseteq G_{i}$ for each $i \in I$; then $L_{C_{1}}^{\prime}$ contains a power $A^{l}$ of a perod $A$. Let $X \in L_{C_{1}}$. Then by the definition of a generating mapping on $\Omega$, there are $W_{i_{1}}, \ldots, W_{i_{1}} \in L_{C_{1}}^{\prime}, t \geq 1$, such that $F(\{X\}) \subseteq F\left(\left\{A^{l}, W_{i_{1}}, \ldots, W_{i_{r}}\right\}\right)$ and for each $s, 1 \leq s \leq t, W_{i_{s}}$ is a minimal word in $G$ not belonging to $\mathrm{gp}\{A\}$. Repeating the proof of assertion 5 of Theorem A, we obtain that $X \in L_{C_{1}}^{\prime}$ and $L_{C_{1}} \leq L_{C_{1}}^{\prime}$.

Assertions 8 and 10 of Theorem A follow from Lemma 3.

It remains to prove that $L$ is simple. Let $M$ be an arbitrary normal subgroup of $L$. If $M$ is a proper subgroup, then we can assume that either $M$ is a subgroup of some group $G_{i}, i \in I$, or $M=\operatorname{gp}\left\{A^{\prime}\right\}$, where $A$ is a period, or $M=R_{C}$, where $C \nsubseteq G_{i}$ for each $i \in I$. We consider these cases.

(1) If $M$ is a subgroup of some group $G_{i}, i \in I$, then there is $Z \in L \backslash G_{i}$, with $Z M Z^{-1}=M$, contradicting [10, Lemma 34.11].

(2) If $M=\operatorname{gp}\left\{A^{t}\right\}$, then there is $Z \in L \backslash \operatorname{gp}\{A\}$ such that $Z M Z^{-1}=M$ contradicting [10, Lemma 34.9].

(3) If $M=R_{C}$, where $C \nsubseteq G_{i}$ for each $i \in I$, then there is $Z \in L$ such that 
$F(\{Z\}) \nsubseteq f(C)$. The group $M$ contains an element $A^{t}$, where $A$ is a period; hence by Lemmas 3 and $1, Z A^{\prime} Z^{-1}$ is not contained in $M$, and we arrive at a contradiction to the choice of the group $M$.

Thus $L$ is simple, and the proof of Theorem A is complete.

\section{Acknowledgments}

This work was done while the author held an Ethel Raybould Fellowship in the Department of Mathematics at the University of Queensland. His visit to Queensland was also supported by an ARC grant.

The author is cordially grateful to Sheila Oates-Williams and Sidney A. Morris for the organization of his visit to Australia, helpful discussions and their encouragement.

\section{References}

[1] S. G. Ivanov, 'Standard and dually standard elements of the lattice of subgroups of a group', Algebra i Logika 8 (1969), 440-446 (translation: Algebra and Logic 8 (1969) 253-256).

[2] S. V. Ivanov, 'Two remarks on groups of finite period', in: 19th All-Union Algebraic Conf., Res. Comm. Part 2, L'vov (1987), 105.

[3] L. S. Kazarin and L. A. Kurdachenko, 'Conditions for finiteness and factorization in infinite groups', Uspekhi Mat. Nauk 47 (1992), 75-114; (English translation: Russian Math. Surveys 47 1992, 81-126).

[4] Kourovka Notebook: unsolved problems of group theory, 12th edition (Inst. Math. Siberian Dep. Russian Acad. Sci., Novosibirsk, 1992).

[5] T. Matumoto, 'Any group is represented by outer automorphism group', Hiroshima Math. J. 19 (1989), 209-219.

[6] F. Napolitani, 'Sui gruppi risolubili complementati', Rend. Sem. Mat. Univ. Padova 38 (1967), $118-120$.

[7] V. N. Obraztsov, 'An embedding theorem for groups and its corollaries', Mat. Sb. 180 (1989), 529-541 (English translation: Math. USSR-Sb. 66 1990, 541-553).

[8] — , 'Embedding schemes for groups and some applications', deposited VINITI 8 February 1990, no. 724-B90.

[9] - 'On infinite complete groups', Comm. Algebra 22 (1994), 5875-5887.

[10] A. Yu. Ol'shanskii, Geometry of defining relations in groups (Nauka, Moscow, 1989); English translation: (North Holland, Amsterdam, 1991).

[11] S. Stonehewer and G. Zacher, 'Dual-standard subgroups in nonperiodic locally soluble groups', Atti. Accad. Naz. Lincei Rend. Cl. Sci. Fis. Mat. Natur. (9) Mat. Appl. 1 (1990), 101-104.

[12] G. Zappa, 'Sulla condizione perche un emitropismo inferiore tipico tra due gruppi sia un omotropismo', Giorn. Mat. Battaglini (4) 80 (1951), 80-101. 
Faculty of Mathematics and Physics

Kostroma Pedagogical University

First of May 14, Kostroma 156601

Russia

Current address:

Department of Mathematics

University of Melbourne

Parkville VIC 3052

Australia

e-mail: vobrazts@maths.unimelb.edu.au 